\title{
USING POINT SYMMETRY IN FINITE ELEMENT ANALYSES
}

\author{
Ştefan SOROHAN ${ }^{1}$, Dan Mihai CONSTANTINESCU ${ }^{1}$, Marin SANDU ${ }^{1}$, Adriana SANDU ${ }^{1}$ \\ 1 University POLITEHNICA of Bucharest, Department of Strength of Materials, Splaiul Independeţei \\ nr. 313, 060042 Bucharest, Romania. E-mail: stefan.sorohan@upb.ro
}

\section{Introduction}

Point symmetry is a rotational symmetry. A half of a part (see Fig. 1), which is rotated by $180^{\circ}$ around an axis $(\mathrm{O} z$ in this paper), generates the complete part. Sometimes it is called origin symmetry. It falls into the large category of cyclic symmetry where the numbers of sectors are only two. When the point symmetry is present, almost all papers in the literature neglect its effect; part of them consider only the reflective symmetry (Zplane Sym) in analyzing some problems which fall into the category of the ones presented in Fig. 2. Only few papers, as [1] consider this type of symmetry but in an approximate manner. A recent work [2] briefly presented the advantages of using point symmetry for $2 \mathrm{D}$ analysis of a single lap joint under tensile load and pointed the basic conditions of constraint equations (CEs) which must be considered.

Commercial finite element codes, as ANSYS, may be used to efficiently solve the cyclic symmetry, but for this particular case, due to the methodology of implementation - as the basic sector is duplicated, considering the cyclic symmetry does not assure a beneficial computational effort.

This paper presents the conditions in which this type of symmetry may be used in finite element modeling to solve a large class of usual problems especially for linear and nonlinear static analysis.

\section{Methodology}

Let us consider a part as in Fig. 1a, which is also point symmetric in material properties and loads. An arbitrarily point symmetric $C^{0}$ surface, or a simple plane $\Sigma$ cuts the part into two parts $\mathrm{A}$ and $\mathrm{B}$ (Fig. 1b-f). A point $A$ in Part A has an associated point symmetric - point $B$ in Part B (Fig. 1c). If the surface $\Sigma$ is a plane, we can define an angle $\alpha$ (Fig. 1d) between this plane and the reference plane Oxz.

Due to the point symmetry conditions, the applied external forces $\boldsymbol{F}$ and moments $\boldsymbol{M}$, in the global system of reference $\mathrm{O} x y z$, must obey the conditions

$$
\begin{aligned}
& F_{x}{ }^{B}=-F_{x}{ }^{A} ; \quad F_{y}{ }^{B}=-F_{y}{ }^{A} ; \quad F_{z}{ }^{B}=F_{z}{ }^{A} ; \\
& M_{x}^{B}=-M_{x}^{A} ; \quad M_{y}^{B}=-M_{y}^{A} ; \quad M_{z}^{B}=M_{z}^{A} .
\end{aligned}
$$

The degrees of freedom - displacements $u$ and rotations $\varphi$ in the parts A and B (or points $A$ and $B$ ) will result in the form

$$
\begin{aligned}
& u_{x}^{B}=-u_{x}^{A} ; \quad u_{y}^{B}=-u_{y}^{A} ; \quad u_{z}^{B}=u_{z}^{A} ; \\
& \varphi_{x}^{B}=-\varphi_{x}^{A} ; \quad \varphi_{y}^{B}=-\varphi_{y}^{A} ; \quad \varphi_{z}^{B}=\varphi_{z}^{A} .
\end{aligned}
$$

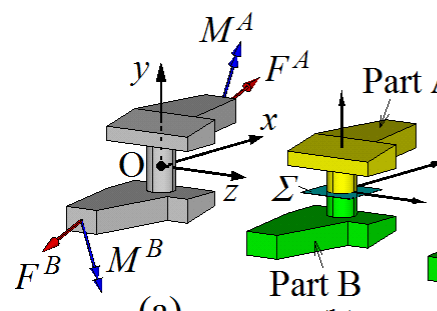

(a)

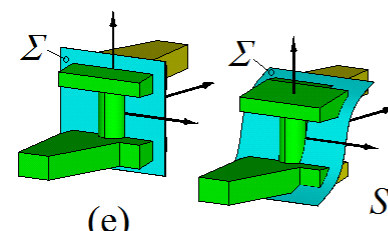

(f)

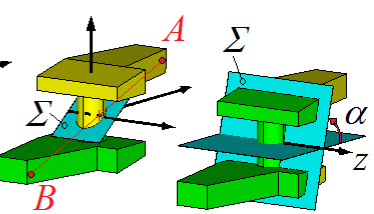

(c)

(d)

Constraint equations

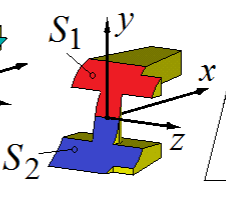

(g)

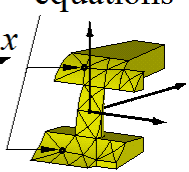

(h)
Fig. 1. Definition of a point symmetry part and some of its possible finite element models.

Also, the stresses will satisfy the conditions:

$$
\begin{aligned}
\sigma_{x}^{B}=\sigma_{x}^{A} ; & \sigma_{y}^{B}=\sigma_{y}^{A} ; & \sigma_{z}^{B}=\sigma_{z}^{A} ; \\
\tau_{x y}^{B}=\tau_{x y}^{A} ; & \tau_{y z}^{B}=-\tau_{y z}^{A} ; & \tau_{x z}^{B}=-\tau_{x z}^{A} .
\end{aligned}
$$

If we keep in an analysis only Part A, the conditions (2) are valid on the points of the surfaces $S_{1}$ and $S_{2}$ in Fig. 1g. This implies that in the finite element model the mesh on these two surfaces must be also point symmetric and Eqs. (2) define the 
constrained matrix between two-point symmetric nodes in the next equation

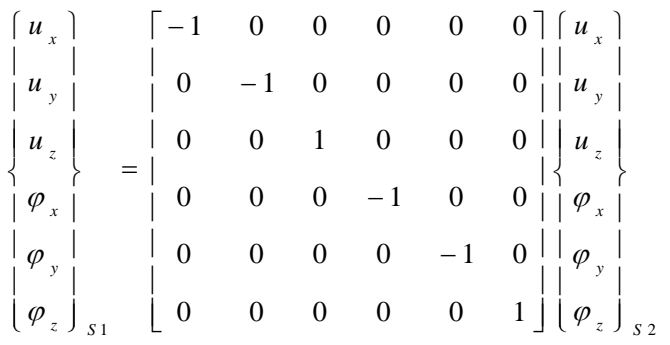

For the common points of the surfaces $S_{1}$ and $S_{2}$, i.e. $\mathrm{O} z$ axis, using Eq. (4) at limits, it yields:

$$
\begin{array}{ll}
u_{x}=0 ; & \varphi_{x}=0 ; \\
u_{y}=0 ; & \varphi_{y}=0,
\end{array}
$$

In Fig. 2, some examples of possible application of point symmetry are presented: tapered single lap joint (a); wavy lap joint (b); reinforced scarf joint (c); rivet-bonded joint (d); reverse bent and boltedbonded joints (e); symmetric lap test specimen (f); sandwich core specimen tested in compression $(\mathrm{g})$. The red and blue surfaces correspond to the surfaces $S_{1}$ and $S_{2}$ where the conditions (4) and (5) must be applied. Using these conditions four rigid body motions are removed.

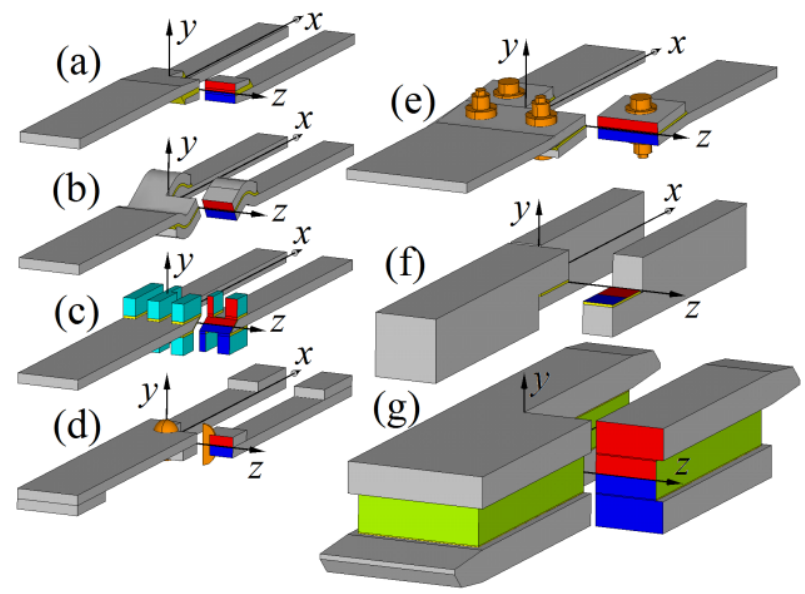

Fig. 2. Examples of point symmetric problems, where the Z-plane Sym exists and the quarter models that may be considered in the analyses.

It must be mentioned that CEs (4) are valid also for nonlinear analyses for large deflections.

\section{An application and results}

A plane shear specimen of a honeycomb core 1/4-5056-0.0025 (5.2 pcf) [3] in a tension test was analyzed using two different models - Model 1 and Model 2. Model 1, neglecting the adhesive between the cell walls, was meshed using only quadrilateral shell elements SHELL181 (6 DOFs/node) which include also in an approximate way the facesheet adhesive. Model 2 was meshed only with solid tetrahedral elements SOLID187 (3 DOFs/node) and replicates all the geometry details including the adhesive between all cell walls and facesheet adhesive with fillets. For both models full, half and quarter models, taking into consideration the reflective and point symmetry, were considered for static linear and large displacement nonlinear analyses. The main results: total number of active DOFs; memory RAM allocated by the code; elapsed time spent for computing the solution only for linear static analyses CP are presented in Table 1 and Table 2. The conditions (4) and (5) were imposed by an APDL file, developed by the authors of this paper.

Table 1. Results for Model 1 in ANSYS Classic.

\begin{tabular}{|r|c|c|r|}
\hline \multicolumn{1}{|c|}{ Model } & $\begin{array}{c}\text { DOFs } \\
{[-]}\end{array}$ & $\begin{array}{c}\text { RAM } \\
{[\mathbf{G B}]}\end{array}$ & \multicolumn{1}{c|}{$\begin{array}{c}\text { CP } \\
{[\mathbf{s e c}]}\end{array}$} \\
\hline Full & 866,232 & 4.233 & 52.4 \\
\hline Half Z-Sym & 433,116 & 1.694 & 20.6 \\
\hline Quarter $\alpha=0^{\circ}$ & 216,554 & 0.791 & 9.8 \\
\hline Quarter $\alpha=90^{\circ}$ & 216,554 & 0.761 & 10.3 \\
\hline
\end{tabular}

Table 2. Results for Model 2 in ANSYS Workbench.

\begin{tabular}{|r|r|r|r|}
\hline \multicolumn{1}{|c|}{ Model } & $\begin{array}{c}\text { DOFs } \\
{[-]}\end{array}$ & $\begin{array}{c}\text { RAM } \\
{[\text { [GB] }}\end{array}$ & $\begin{array}{c}\text { CP } \\
{[\mathbf{s e c}]}\end{array}$ \\
\hline Full & $4,101,997$ & 8.065 & 1177.7 \\
\hline Half Z-Sym & $2,066,299$ & 4.122 & 1032.4 \\
\hline Quarter $\alpha=0^{\circ}$ & $1,038,792$ & 7.598 & 54.7 \\
\hline Quarter $\alpha=90^{\circ}$ & $1,042,431$ & 7.734 & 67.3 \\
\hline
\end{tabular}

\section{Conclusions}

If we consider as reference the well known half reflective Z-Sym model, the additional consideration of the point symmetric model, i.e. quarter model in this paper, may reduce the computational effort with minimum $50 \%$, or even more, depending on the size of the models and the used computer, or the finite element code. For example, considering the presented application and Model 2, the CP reduces almost 20 times $(1032.4 / 54.7=18.87)$.

\section{References}

[1] Tsai, M.Y., Morton, J. Three-dimensional deformations in a single-lap joint. J Strain Anal Eng, 1994, 29(1), 137-145.

[2] da Silva, L.F.M., Öchsner, A. (Eds.). Modeling of Adhesively Bonded Joints; Springer-Verlag: Berlin Heidelberg, 2008.

[3] HexWeb ${ }^{\mathrm{TM}}$, Hexcel Corporation, 1999. https://www.hexcel.com. Accessed February 07, 2018. 